\title{
A TÉCNICA DE LICHTENSTEIN NAS HÉRNIAS INGUINAIS PRIMÁRIAS E RECIDIVADAS - CIRURGIA AMBULATORIAL EM HOSPITAL UNIVERSITÁRIO
}

\section{Lichtenstein hernia repair in primary and recurrent inguinal hernias - ambulatory surgery in a university hospital}

\author{
Marco Antonio de Oliveira PERES, Tâmara Maria NIERI, \\ Heitor Sebastião de BARCELOS NETO, Nelson Adami ANDREOLLO
}

ABCDDV/563

Peres MAO, Nieri TM, Barcelos Neto HS, Andreollo NA. A técnica de Lichtenstein nas hérnias inguinais primárias e recidivadas - cirurgia ambulatorial em hospital universitário. ABCD Arq Bras Cir Dig 2007;20(4):221-4.

RESUMO - Racional - A técnica de Lichtenstein é o padrão-ouro na cirurgia das hérnias ingui-nais, permitindo fixação de prótese de polipropileno, sem tensão na linha de sutura, baixa recidiva, ampla aplicabilidade, fácil ensino aos jovens cirurgiões, alta precoce e menores custos. Objetivo - Análise retrospectiva dos resultados na cirurgia ambulatorial das hér-nias inguinais primárias e recidivas pela técnica de Lichtenstein em Hospital Universitário. Métodos - Entre agosto de 1994 a dezembro de 2001 foram realizadas 343 hernioplastias inguinais em 326 portadores de hérnias inguinais primárias ou recidivadas. Eram $304(88,6 \%)$ masculinos, com idade que variou de 19 a 85 anos. Doenças concomitantes fo-ram observadas em 196 casos (60,1\%), predominando hipertensão, tabagismo e cardio-patia. Quanto ao lado, 232 apresentavam-se à direita e 94 à esquerda; dezessete doen-tes $(7,3 \%)$, todos homens, tinham apresentação bilateral. Dentre as $38(11,1 \%)$ hérnias recidivadas, 27 eram do lado direito. A técnica clássica de Lichtenstein foi realizada com prótese de polipropileno, de 15 × 7,5 cm, fixada com fio do mesmo material. Resultados - A anestesia local foi a primeira escolha em $55,9 \%$. Em apenas um caso $(0,3 \%)$ foi neces-sária a intervenção do anestesista e a conversão da anestesia local para geral. Quatro doentes necessitaram permanência hospitalar de um dia. As complicações locais preco-ces registradas foram de seroma: 15 casos; infecção superficial de ferida operatória: 11 (3,3\%); hematoma: 5 casos; e trombose venosa de cordão espermático: 2 casos. No se-guimento tardio de cinco anos, a recidiva da hérnia foi observada em três casos $(0,87 \%)$. Conclusão - Esta técnica revelou ser de fácil aplicação, segura, eficiente, podendo ser realizada sob anestesia local e ambulatorial, com baixos índices de complicações e baixa taxa de recidiva. Permitiu o ensino de alunos e residentes, bem como atender à demanda reprimida desta doença tão freqüente nos ambulatórios.

DESCRITORES - Hérnia inguinal. Técnica de Lichtenstein. Cirurgia ambulatorial. Anestesia local.

\section{INTRODUÇÃO}

A hérnia inguinal é afecção muito prevalente com impacto sócio-econônimo relevante no mundo ${ }^{13} \mathrm{e}$ também no nosso meio ${ }^{20,23}$. O tratamento recomendado é o cirúrgico o qual tem passado por importantes avanços nas últimas duas décadas, com destaque nas estatísticas mostrando queda substancial das taxas de recorrência da doença, assim como nos baixos relatos recentes de cirurgias para hérnias recidivadas. Fator determinante neste progresso foi a rotina de implantação de prótese ou tela de polipropileno na região ínguino-crural, quer por acesso anterior via inguinotomia, quer por acesso posterior na via laparoscópica ${ }^{11,26}$.

A primeira vez que foi utilizado na literatura o termo "hernioplastia isenta de tensão" ocorreu em 1986, por Lichtenstein et a ${ }^{18}$. Descreveram com detalhes a técnica que utiliza, por inguinotomia, a tela de polipropileno suturada sobre a fáscia transversal, que por si só representa o reparo

Trabalho realizado no Setor de Hérnias do Programa de Cirurgia Ambulatorial do Departamento de Cirurgia da Faculdade de Ciências Médicas da Universidade Estadual de Campinas - UNICAMP, Campinas - SP

Endereço para correspondência: Marco Antonio de Oliveira Peres, e-mail: marcoperes@terra.com.br real e que se aplica em todos os tipos de hérnias inguinais diretas ou indiretas. Esta técnica rapidamente atingiu o "padrão-ouro" no tratamento das hérnias inguinais devido à característica de ser eficiente, de ampla aplicabilidade, de fácil ensino aos cirurgiões mais jovens, de custos não elevados e podendo ser realizada sob anestesia local em sistema ambulatorial ${ }^{2,4,9,11,13,22}$.

A minuciosa aplicação da técnica, como descrita com detalhes pelo autor, tem sido o alicerce dos melhores resultados tardios, com recorrências menores que 1\% descritos na literatura ${ }^{7,8,18}$. Enfatiza-se que a maioria dos insucessos no Lichtenstein ocorre por falha técnica do executante, geralmente no início de suas atividades ${ }^{4,17}$. As suas vantagens também incluem tratamento bilateral no mesmo ato sem prejuízo dos resultados tardios e a recuperação das atividades laborativas sem esforços em menor tempo. Além disso, não restringe o paciente das suas diversas atividades com maiores esforços após período de dez semanas.

Centros de ensino, faculdades e universidades adotaram rapidamente a técnica de Lichtenstein pela sua eficácia. Porém, muitos destes grandes serviços concentram suas atividades como função de hospital terciário, não 
disponibilizando leitos, salas cirúrgicas e anestesistas para programas de tratamento de algumas doenças, como as hérnias. Neste contexto, a fim de evitar falha no ensino de alunos e residentes em cirurgia geral, o Departamento de Cirurgia e o Hospital das Clínicas da Faculdade de Ciências Médicas da UNICAMP incrementaram o Programa de Cirurgia Ambulatorial em centro cirúrgico anexo, com toda estrutura para alta no mesmo dia, permitindo avanços no tratamento das hérnias e grande benefício aos doentes e a toda comunidade da área de referência deste hospital, além da redução de custos. Outras séries relatam até $26,5 \%$ de economia nos gastos hospitalares quando comparados aos doentes internados ${ }^{28}$.

O objetivo deste trabalho foi análise retrospectiva dos resultados tardios do tratamento cirúrgico das hérnias inguinais primárias e recidivas pela técnica de Lichtenstein, ambulatorialmente, conforme protocolo préestabelecido, no Hospital das Clínicas (HC) da UNICAMP.

\section{MÉTODOS}

Os doentes referenciados para consultas no Ambulatório de Hérnias do HC, foram incluídos no Programa de Cirurgia Ambulatorial respeitando os seguintes critérios de inclusão: doentes de ambos os sexos, com idade maior que 18 anos, portadores de hérnias inguinais redutíveis, aceitação da alta no mesmo dia, com estrutura familiar mínima para trazer e levar do hospital e cumprir as orientações no domicílio, residência próxima de $100 \mathrm{Km}$ ou 1 hora de traslado até a instituição. Foram excluídos deste protocolo doentes com hérnias crurais, hérnias irredutíveis, mais do que duas cirurgias para recidivas no mesmo local, obesos, doenças concomitantes de difícil controle, descrédito no programa, antecedente de alcoolismo, convulsões, histeria e de distúrbios psiquiátricos. Os portadores de hérnias bilaterais foram indicados para a realização da operação sob anestesia local de um lado, escolhendo primeiro o lado mais limitante, e postergando-a do outro lado entre dois e três meses após. A exceção para realizar cirurgia bilateral concomitante foi mediante consenso com os próprios doentes provenientes de regiões mais distantes e que não teriam a chance de retornar mais ao serviço meses após, sendo nestes casos optado pela anestesia por bloqueio lombar.

Os exames complementares pré-operatórios indicados foram em número reduzido, sendo: hemograma, glicemia e coagulograma de rotina; eletrocardiograma acima dos quarenta anos ou qualquer idade se doença cardiovascular prévia; e eventuais exames específicos de doenças crônicas em tratamento, como pneumopatia, prostatismo e coagulopatia.

Assim, no período compreendido entre agosto de 1994 a dezembro de 2001 foram realizadas 343 hernioplastias inguinais pela técnica de Lichtenstein em 326 doentes portadores de hérnias inguinais primárias ou recidivadas. Quanto ao sexo, eram $304(88,6 \%)$ do masculino e 22 $(11,4 \%)$ do feminino. A idade variou de 19 a 85 anos, com mediana aos 62 anos. Doenças concomitantes foram observadas em 196 doentes $(60,1 \%)$, sendo mais freqüentes a hipertensão, tabagismo e cardiopatia. Quanto ao lado, 232 apresentavam-se unicamente à direita e 94 somente à esquerda. Dezessete casos (7,3\%), todos homens, tinham apresentação bilateral. Dentre as $38(11,1 \%)$ operações para hérnias recidivadas, 27 eram do lado direito.

A técnica clássica de Lichtenstein foi indicada em todos os doentes utilizando prótese de polipropilene, de $15 \times 7,5$ $\mathrm{cm}$, fixada com fio do mesmo material (polipropileno 2-0). As hérnias diretas ou recidivadas tiveram reduzidos seus abaulamentos pela reconstrução da fáscia transversal antes da fixação da tela. Hérnias indiretas tiveram, além disso, seus sacos herniários ressecados após ligadura alta.

A anestesia local foi a primeira escolha nas hérnias inguinais, reservando o bloqueio lombar para as hérnias ínguino-escrotais maiores, doentes não magros, ansiosos e com alguma doença de base mais lábil.

Os doentes indicados para anestesia local, tiveram jejum absoluto confirmado de oito horas, tricotomia local, monitorização com cardioscópio e oxímetro de pulso, venóclise obrigatória durante todo o ato operatório, sedação endovenosa com midazolan de 3 a $5 \mathrm{mg}$ associado a meperidina $30 \mathrm{mg}$, sendo obrigatório a presença de anestesista nas imediações. A anestesia local da região seguiu o modelo de Ponka ${ }^{24}$ e Amid ${ }^{1}$, realizado pelo próprio cirurgião, também chamada de bloqueio de campo ${ }^{25}$, utilizando uma cuba com $80 \mathrm{~mL}$ de solução, sendo $20 \mathrm{~mL}$ de lidocaína a $1,0 \%$ sem vasoconstritor misturada com 20 $\mathrm{mL}$ de bupivacaína a $0,25 \%$ com vasoconstritor e acrescidos $40 \mathrm{~mL}$ de solução salina a $0,9 \%$, como em trabalho anterior bem sucedido da equipe ${ }^{5}$. A anestesia raquídea, peridural ou geral quando indicada foi realizada pela equipe de anestesistas. Não foi indicado antibioticoterapia de rotina, salvo indicação prévia.

Os critérios de alta obedecidos foram: período de recuperação mínimo de quatro horas para os operados sob anestesia local e o tempo maior determinado pelo anestesista quando foi realizado bloqueio lombar. Além disso, foram verificados rigorosamente os registros de dados vitais estáveis, controle de dor e sangramento na ferida operatória, razoável aceitação alimentar sem vômitos, apresentar ao menos uma diurese espontânea, e receber todas as orientações pós-operatórias verbais e por escrito junto com seu acompanhante adulto, tais como retorno no ambulatório, telefones para dúvidas e local de procura na instituição em eventual emergência e a confirmação do transporte. Os retornos foram programados para: primeiro entre 7 a 10 dias, visando a retirada dos pontos; depois no $1^{\circ}, 2^{\circ}, 6^{\circ}$ meses e uma vez por ano até $5^{\circ}$ ano.

\section{RESULTADOS}

Conforme a classificação de Nyhus $^{21}$, 11 doentes da casuística pertenciam ao tipo II (indiretas); 138 ao tipo IIIA (diretas); 156 ao tipo IIIB (mistas escrotais); e 38 ao tipo IV (recidivadas). Doze doentes com hérnias bilaterais foram operados de um lado de cada vez em épocas diferentes sob anestesia local e cinco doentes com hérnias bilaterais tiveram operação concomitante sob bloqueio lombar. 
A anestesia empregada foi a local em 192 (55,9\%) procedimentos; peridural em 115 (33,5\%), geral em 27 $(7,9 \%)$, raquídea em $7(2 \%)$ e peridural associada à geral em $2(0,6 \%)$. Foi opção dos anestesistas indicarem o melhor método para o doente, quando não foi possível anestesia local. Em apenas um caso $(0,3 \%)$ foi necessário a intervenção do anestesista e a conversão da anestesia local para anestesia geral, devido a incontrolável agitação psicomotora durante o ato cirúrgico. Antibióticos não foram utilizados em 291 casos $(84,8 \%)$. Tiveram alta hospitalar, no mesmo dia, 322 doentes $(98,8 \%)$. Os quatro doentes que necessitaram permanência hospitalar até a manhã seguinte foram por complicações gerais, sendo: bradicardia sintomática em dois casos, crise hipertensiva em um caso e retenção urinária em um caso. Dois doentes tiveram lipotímia e hipotensão arterial logo após a alta da unidade, sendo medicados e liberados a seguir. Além disso, outro doente apresentou flebite superficial em membro superior.

As complicações locais precoces registradas foram: seroma em 15 casos, infecção superficial de ferida operatória em 11 (3,3\%), hematoma em 5 casos e trombose venosa de cordão espermático em 2 casos. Em um caso foi identificada "missing" hérnia crural no $4^{\circ}$ dia pósoperatório com obstrução de intestino delgado, e o doente foi operado utilizando plugue de polipropileno em forma de rolete á Lichtenstein, com sucesso.

Por meio de consulta dos prontuários, foram obtidas informações de 269 (82,5\%) doentes com seguimento tardio até cinco anos de pós-operatório e a recidiva da hérnia foi constatada em 3 casos $(0,87 \%)$; dor crônica tratada pelo grupo de dor do hospital sem necessidade de reintervenção em dois, hidrocele em um e granuloma por fio de sutura em um. Dentre os três casos de recidivas, duas ocorreram próximas ao sexto mês e uma pouco mais de um ano após $o$ retorno às atividades laborativas. Os doentes foram reoperados pelo grupo, sendo constatado falha técnica da fixação da tela de polipropileno junto ao púbis.

\section{DISCUSSÃO}

Os hospitais universitários, terciários, públicos e de ensino, tem apresentado dificuldades na realização de procedimentos cirúrgicos de nível de complexidade I e II, sendo este último o que detém a doença herniária. Não existem leitos específicos para as hérnias nestes nosocômios, há escassez de horários disponíveis em centro cirúrgico e de anestesistas em detrimento de doenças de complexidade terciária, como neoplasias, traumas e transplantes.

Deste modo, duas opções se impõem: implantação de um programa de cirurgia ambulatorial em centro cirúrgico anexo específico ou em hospitais secundários com convênios universitários. Durante sete anos a UNICAMP não teve associação com outro hospital, exigindo, portanto a escolha da primeira opção para atender à comunidade. As vantagens da implantação do referido programa foram grandes, incluindo a assistência à comunidade e o ensino de residentes de cirurgia geral e alunos de graduação do curso médico.

A implantação da técnica de Lichtenstein em nosso serviço universitário, portanto de ensino, trouxe resultados animadores $^{23}$, sendo considerada a mais adequada, pela eficiência e aplicabilidade na proposta ambulatorial e de baixo custo $^{28}$. Foram respeitados os detalhes técnicos originais, sem modificações, como preconizado por $\mathrm{Amid}^{2}$. Não foi difícil reproduzir o que vários serviços cirúrgicos no mundo vinham realizando ${ }^{3,15}$, com sucesso e economia, incluindo enfermagem, assistentes sociais e pessoal de apoio para a liberação precoce do doente para o seu domicílio ${ }^{16}$. A anestesia local estava padronizada, com riscos mínimos e baixíssimas taxas de conversão para outros métodos ${ }^{5,24,29}$.

No nosso país alguns autores enfatizaram a utilização da anestesia local, associada à sedação, para uma considerável parcela dos pacientes submetidos à operação de hérnias inguinais ${ }^{12,19,20}$. Bloqueio lombar, quer raquídeo ou peridural já tinham suas rotinas e drogas para a alta no mesmo dia. Apenas um caso teve necessidade de convocar o anestesista para conversão de anestesia local para geral por agitação, sem confirmação se a causa era psicogênica ou pela circulação de anestésicos, já que não teve hipoxemia. Alguns autores referem que cerca de $1 \%$ dos submetidos à herniorrafia ambulatorial necessitarão hospitalização prolongada ${ }^{10}$. Porém, o ensinamento adquirido com este caso foi a necessidade da presença constante de anestesista no setor.

Quanto à avaliação e preparo pré-operatório os exames complementares foram em número reduzido, diminuindo portanto o custo geral do tratamento cirúrgico. Muitos dos próprios doentes, por conhecimento de parentes, amigos a até a mídia, tem solicitado a colocação de tela ou prótese, por se sentirem mais seguros e com confiança no resultado tardio da operação, o que é rotina do serviço. A maioria deles demonstraram interesse na alta hospitalar no mesmo dia, pelas vantagens de menor alteração de sua rotina de vida pessoal e dos familiares. Assim, a análise dos resultados mostrou que apenas quatro casos $(1,2 \%)$ necessitaram de internação, sendo que dois tinham recebido anestesia local e dois por bloqueio.

Especificamente quanto ao ato cirúrgico, não há diferença da aplicação de técnica de Lichtenstein quando realizada por anestesia local, bloqueio ou geral, nem aumento do tempo operatório, em acordo com outras séries $^{20,28}$. Além disso, não foi detectada dificuldade maior se a hérnia inguinal era recidivada uma ou duas vezes, conforme o protocolo neste estudo, pois em nenhuma das operações anteriores tinham sido colocadas telas. Nos doentes com múltiplas recidivas ou com prévia colocação de tela, não considerou-se oportuno indicar o método ambulatorial e nem a anestesia local, pelo maior risco de complicações e insucessos.

Nesta casuística, os três casos de recidiva registrados no seguimento ambulatorial tiveram, como etiologia, defeito técnico na fixação da tela a nível do púbis, detalhe este considerado muito importante e que recebeu atenção maior na época, tanto que nos últimos cinco anos não 
foram mais registradas outras recidivas neste local. As recidivas comentadas na literatura também são atribuídas a erro técnico em fase inicial de aprendizado da equipe, geralmente a nível da púbis ${ }^{3,6,27}$.

\section{CONCLUSÃO}

A técnica de Lichtenstein no tratamento das hérnias inguinais primárias e recidivadas revelou ser de fácil aplicação, segura, eficiente, podendo ser realizada sob anestesia local e alta no mesmo dia, com baixos índices de complicações e baixa taxa de recidiva. Permitiu o ensino de alunos e residentes da técnica considerada "padrão-ouro" de tratamento, bem como atender à demanda reprimida desta doença tão freqüente nos ambulatórios.

Peres MAO, Nieri TM, Barcelos Neto HS, Andreollo, NA. Lichtenstein hernia repair in primary and recurrent inguinal hernias - ambulatory surgery in a university hospital. ABCD Arq Bras Cir Dig 2007;20(4):221-4.

ABSTRACT - Background - Lichtenstein hernia repair is gold standard in inguinal hernia sur-geries, allowing a tension-free polypropylene mesh, low recurrence, wide applicability, easy teaching to young surgeons, early discharge and lower costs. Aim - A retrospective analysis was made using the results in the ambulatory surgery of primary inguinal hernias and recurrences, performing the Lichtenstein technique in a University Hospital. Methods - From August of 1994 to December of 2001, 343 inguinal hemioplasties were performed in 326 patients with primary and recurrent inguinal hernias. Three hundred and four (88.6\%) were male, with ages ranging between 19 to 85 years. Concomitant diseases were ob-served in 196 cases $(60.1 \%)$, were hypertension, smoking and cardiopathy were predomi-nant. Regarding the side of the hernias, 232 were on the right and 94 on the left side; sev-enteen male patients (7.3\%) showed bilateral presentation. Among the $38(11.1 \%)$ recur-rent hernias, 27 were on the right side. The classic Lichtenstein technique was indicated in all, using polypropylene mesh, of $15 \times 7.5 \mathrm{~cm}$. Results - Local anesthesia was the first choice in $55.9 \%$ of cases. Anesthetist intervention and the conversion of the local anesthe-sia for general was necessary in only one case $(0.3 \%)$. Four patients needed single day hospital admissions. Early local complications were of seroma: 15 cases; superficial infec-tion of operative wound: 11 (3.3\%); hematoma: 5 cases and venous thrombosis of the spermatic cord : 2 cases. After a five year follow-up, hernia recurrences were observed in three cases (0.87\%). Conclusion - The technique revealed to be of easy application, could be performed under local anesthesia and as an outpatient, with low rates of compli-cations and recurrences. It also allowed students and residents to learn the technique, as well as to attend to such demanding and frequent disease in daily clinics.

HEADINGS - Inguinal hernia. Lichtenstein technique. Ambulatory surgery.

\section{REFERÊNCIAS}

1. Amid PK, Shulman AG, Lichtenstein IL. Local anesthesia for inguinal hernia repair step-by-step procedure. Ann Surg 1994; 220(6):735-7.

2. Amid PK. Lichtenstein tension-free hernioplasty: its inception, evolution and principles. Hernia 2004; 8:1-7.

3. Amid PK. The Lichtenstein repair in 2002: an overview of causes of recurrence after Lich-tenstein tension-free repair. Hérnia 2003; 7:13-6.

4. Balen EM, Ferrer JV, Vicente F, Blazquez L, Herrera J, Lera JM. Recurrences after pros-thetic repair of inguinal hernias by the Lichtenstein technique. Hernia 2000; 4:13-6.

5. Barcelos Neto HS, Peres MAO, Labbate DV, Seabra JCT, Oliveira C, Moraes G, Aranha NC, Barreto G, Leonardi LS. Tratamento cirúrgico das hérnias ínguino-crurais com a-nestesia local - análise de 442 procedimentos. Rev Bras Cir 1996; 86(3): 121-6.

6. Bay-Nielsen M, Nordin P, Nilsson E, Kehlet H. Operative findings in recurrent hernia af-ter a Lichtenstein procedure. Am J Surg 2001;182:134-6.

7. Benfatto G, Catania G, D’Antoni S, Benfatto S, Licri V, Basile G, Tenaglia L. Recurrence after hernioplasty according to Lichtenstein: analysis of the cause. G Chir 2002; 23:427-30.

8. Bisgaard T, Bay-Nielsen M, Christensen, Kehlet H. Risk of recurrence 5 years or more after primary Lichtenstein mesh and sutured inguinal hernia repair. $\mathrm{Br}$ J Surg 2007; 94:1038-40.

9. Chastan P. Tension-free inguinal hernia repair: a retrospective study of 3000 cases in one center. Int Surg 2005; 90(1):48-52.

10. de Vooght A, Droissart R, Staudt JP. Open mesh plug hernioplasty in ambulatory sur-gery: a stud feasibility based on our experience in 413 procedures. Hernia 2002; 6(3):108-12.

11. Fitzgibbons R. Management of an inguinal hernia: Conventional? Tension-free? Laparo-scopic? or may be no treatment at all. General Session of the American College of Sur-geons. 86th Annual Clinical Congress, 2000.

12. Henriques AL, Pezzolo S, Silva GC. Herniorrafia inguinal sob anestesia local. Rev Col Bras Cir. 1997; 24(6):405-8.

13. Kark AE, Kurzer MN, Belsham PA. Three thousand one hundred seventy-five primary in-guinal hernia repairs: advantages of ambulatory open mesh repair using local anesthe-sia. J Am Coll Surg 1998, 186(4):447-456.

14. Kurzer M, Belsham PA, Kark AE. The Lichtenstein repair for groin hernias. Surg Clin North Am 2003; 83(5):1099-117.

15. Lau H, Lee F, Poon J. Clinical factors influencing return to work after ambulatory inguinal herniorrhaphy in Hong Kong. Ambul Surg. 2001; 9(1):25-8.

16. Laurino Neto RM, Buchmann AACM, Messias LRR. Tratamento cirúrgico das hérnias in-guinais sob anestesia local em ambulatório. Rev Col Bras Cir. 2004; 31(2):102-6.
17. Lichtenstein IL, Shulman AG, Amid PK, et al. The tension-free hernioplasty. Am J Surg 1989; 157:188-93.

18. Lichtenstein IL, Shulman AG. Ambulatory outpatient hernia surgery, including a new con-cept, introducing tension-free repair. Int Surg 1986; 71:1-7.

19. Lima Neto EV, Goldenberg A, Jucá MJ. Resultados imediatos da herniorrafia inguinal com anestesia local associada com sedação. Acta Cir Bras 2003; 18(5):478-84.

20. Lopes AG, Souza JCL, Bienik TS, Velozo TS, Nader PA, Cardoso JR. Tratamento da hérnia inguinal com anestesia local. ABCD Arq Bras Cir Dig 2004; 17(1):26-8.

21. Nyhus LM. Individualization of hernia repair: a new era. Surgery 1993; 114(1):1-2.

22. Pereira JCR, Trugilho JCV, Eulálio JMR, Jamel N. Avaliação do tratamento da hérnia inguinal sob anestesia local e sedação em 1560 pacientes. Rev Col Bras Cir 2006;33(6):375-9.

23. Peres, MAO, Nieri, TM, Barcelos HS, Leonardi LS. Hernioplastia inguinal pela técnica de Lichtenstein nas hérnias inguinais diretas e recidivantes em meio universitário. Rev Col Bras Cir 1999; 26:74-74.

24. Ponka, JL. Seven steps to local anesthesia for inguinofemoral hernia repair. Surg Gyne-col Obstet 1963; jul:115-20.

25. Rodrigues Jr AJ, Mittelstaed WEM, Bevilacqua RG, Birolini D. Anestesia por bloqueio de campo no tratamento ambulatorial das hérnias inguinais. Rev Col Bras Cir 1992; 19(5):195-8.

26. Schwab R, Conze J, Willms A, Klinge U, Becker HP, Schumpelick V. Rezidivleisten-hernienreparation nach vorangegangener Natzimplantation. Chirurg 2006; 77:523-30.

27. Shulman AG, Amid PK, Lichtenstein IL. A survey of non-expert surgeons using the open tension-free mesh repair for primary inguinal hernias. Int Surg 1995;80:35-6.

28. Silva DN, Griebeler ML, Fernandes SF, Paixão LQ, Pitrez FAB. Herniorrafia ambulatorial comparada à convencional. Rev Col Bras Cir 2004; 31(5): 28790.

29. Skinovsky J, Sigwalt MF, Bertinato LP, Chibata M, Moreira LMS, Granzotto PCD. Herni-orrafia inguinal com anestesia locorregional - uso de ropivacaína. Rev Col Bras Cir 2006; 33(4): 224-7.

30. Souza JAG \& Silva A. Cirurgia Ambulatorial. Atheneu, Rio de Janeiro, 1999.
Conflito de interesse: não há

Fonte financiadora: não há

Recebido para publicação em: 23/06/2007

Aceito para publicação em: 04/10/2007 